\title{
Counting of viable C. burnetii cells by quantitative reverse transcription PCR using a recombinant plasmid $(\mathrm{pCB}-\operatorname{dot} \mathrm{A})$ as a standard
}

\author{
F. ZÚÑIGA-NAVARRETE ${ }^{1}$, G. FLORES-RAMIREZ ${ }^{* *}$, M. QUEVEDO-DÍAZ1 ${ }^{1}$ L. ŠKULTÉTY ${ }^{1,2}$
}

${ }^{1}$ Institute of Virology, Biomedical Research Center Slovak Academy of Sciences, Dúbravská cesta 9, 84505 Bratislava, Slovak Republic; ${ }^{2}$ Institute of Microbiology of the CAS, v.v.i., Vídeňská 1083, 14220 Prague, Czech Republic

Received March 22, 2018; revised April 26, 2018; accepted September 25, 2018

\begin{abstract}
Summary. - Coxiella burnetii is an intracellular pathogenic bacterium and etiological agent of Q fever in humans. Recently, the bacterium has been set free from the strictly intracellular condition by successful cultivation in acidified citrate cysteine medium. Here, we report a bacterial cell counting method that allows rapid quantification of the absolute or relative number of live cells of $C$. burnetii in a high throughput manner. The method utilizes TaqMan-based quantitative polymerase chain reaction (qPCR) targeting a single $\operatorname{dot} A$ gene for determination of genome equivalent (GE) presented either as DNA or complementary DNA (cDNA) synthesized via reverse transcription. The assay was shown to be specific, sensitive and efficiently reproducible. The quantification was linear over a range of 30 to $3 \times 10^{8}$ copies. Since there is only one copy of the $\operatorname{dot} A$ gene per Coxiella chromosome, the calculated dotA copy numbers can be compared to the number of bacterial cells. Finally, we demonstrated the potential of the method to assess effects of antibiotic on cell viability and to determine the antibiotic-tolerant fraction within a cell population.
\end{abstract}

Keywords: Coxiella burnetii; Q fever; real-time polymerase chain reaction; copy number; antibiotic; axenic media; $\operatorname{dot}$ A gene

\section{Introduction}

Coxiella burnetii is an intracellular Gram-negative coccobacillus that is a causative agent of Q fever in mammals and humans. The disease is often asymptomatic but under certain circumstances can be manifested as a mild illness similar to influenza with debilitating fever. A small percentage of people who become infected with $C$. burnetii may develop a more serious chronic form of the disease that is usually exhibited as culture-negative endocarditis in patients with previous valvulopathy (Maurin and Raoult 1999). Humans

${ }^{*}$ Corresponding author. E-mail: virugafl@savba.sk; phone: +4212-59302406.

Abbreviations: ACCM = acidified citrate cysteine medium; $\mathrm{AF}=$ antibiotic-free cultivated C. burnetii; $\mathrm{AT}=$ doxycycline-treated culture; $\mathrm{Cq}=$ threshold cycle; $\mathrm{dpi}=$ days post-inoculation; $\mathrm{E}=$ assay efficiency; GE = genome equivalent; HTS = high throughput screening; RT-qPCR = reverse transcription quantitative PCR; $\mathrm{SD}=$ standard deviation of the mean are usually infected by inhalation of aerosols, contaminated by infectious particles derived from domestic animals, particularly after contact with parturient females and their birth products (Babudieri, 1959; Marrie, 1990). Q fever is a zoonosis with a worldwide distribution. Many species of mammals, birds, and ticks are reservoirs of $C$. burnetii in nature. The infection in an animal is most often latent, with persistent shedding of bacteria into the environment. However, in the female reproductive tract, high-level shedding occurs at the time of parturition, with millions of bacteria being released in the placenta.

Treatment with antimicrobial agents is recommended for both acute and chronic $\mathrm{Q}$ fever. For acute $\mathrm{Q}$ fever, a variety of antibiotics have been used, but the member of a tetracycline group, doxycycline, is considered the medicament of choice. Doxycycline has been shown to result in a mean time to defervescence of 2-3 days, whereas untreated patients resolve the fever after a mean of 12.5 days. This antibiotic has shown effectivity also for therapy of chronic form, but it was demonstrated that the combination of doxycycline with chloroquine 
or hydroxychloroquine had much higher bactericidal activity than doxycycline alone (Raoult et al., 1999). It significantly reduced the time of treatment in chronic $\mathrm{Q}$ fever patients to 18 months for those with native heart valves and 24 months for patients with prosthetic valves, which was previously as long as five years. While, resistance to these antibiotics does not appear to be common, natural resistance is still a concern.

C. burnetii has a low rate of duplication. As an obligate intracellular pathogen, it can be cultivated in yolk sacs of embryonated hen's eggs and in various cell lines, including human embryonic fibroblast cells, mosquito cells, L cells or Vero cells (Baca and Paretsky, 1983). However, it has recently been set free from the strictly intracellular conditions with successful cultivation in an acidified citrate cysteine medium (ACCM) (Omsland et al., 2009, 2011). There are several direct and indirect methods for determination of the cell growth and/or viability, each with its characteristic pros and cons. Direct methods include microscopic counting, electronic particle counting, image analysis, in situ biomass monitoring, and dielectrophoretic cytometry. Indirect methods involve the chemical analysis of a culture component or a measurement of metabolic activity. The most common direct method is optical density measurement of bacterial biomass by spectrophotometry. This approach is well suited for high throughput screening (HTS) but does not differentiate between dead and living bacteria. Moreover, the method has a very low sensitivity. Thus, for counting the number of viable cells, a plaque-forming unit (PFU) method seems to be a good choice. Using this method, only the viable infectious particles are counted on the agar overlay, since the inactive particle failing to infect a host cell will not be able to produce a plaque. However, the most important disadvantage of this method is that clumps of bacterial cells can be miscounted as a plaque. In addition, the PFU method is relatively timeconsuming and quite tedious. Thus, it is not well suited for HTS studies (as monitoring of the effect of antibiotics on growth, viability, and apoptosis) (Wike et al., 1972).

Several methods based on polymerase chain reaction (PCR) have been developed for detection and estimation of the bacterial quantity. Nevertheless, also these approaches have some drawbacks. The correlation between cell viability and detection of DNA is problematic due to persistent DNA, which can be present in the medium for a period of time even after cell death (Masters et al., 1994). Thus, the DNA analysis can extremely increase the false-positive rate of the detection (Keer and Birch, 2003). On the other hand, utilization of mRNA as viability marker seems to be more trustworthy because this molecule is more labile and has a shorter half-life after its synthesis inside the cell than DNA (Keer and Birch, 2003). For its utilization, is necessary reverse transcription PCR (RT-PCR), in which the RNA is transcribed to complementary DNA (cDNA), which is then used as a template for quantitative detection of gene expres- sion (Bustin and Nolan, 2009). Herein, we report a bacterial cell counting method that allows rapid quantification of the absolute or relative number of live cells in a bacterial culture in a high throughput manner.

\section{Materials and Methods}

pCB-dotA plasmid construction. A $291 \mathrm{bp}$ fragment from $C$. burnetii Nine Mile $\operatorname{dot} A$ gene was amplified using the primer pair dotA30/31(5'-GGCAATCCCGAAGATGATTA-3' and 5'-CATT GCGCCATCACTTCC-3'). The PCR was performed on a Biometra thermocycler (Analytic Jena Company) as follows: initial denaturation at $95^{\circ} \mathrm{C}$ for $30 \mathrm{~s}, 37$ cycles of denaturation at $95^{\circ} \mathrm{C}$ for $1 \mathrm{~min}$, annealing at $56^{\circ} \mathrm{C}$ for $30 \mathrm{~s}$, and extension at $72^{\circ} \mathrm{C}$ for $30 \mathrm{~s}$ followed by a final extension period at $72^{\circ} \mathrm{C}$ for $5 \mathrm{~min}$. The amplicon was then subcloned into a PCR2.1 TA cloning vector (Invitrogen - ThermoFisher Scientific) and propagated in Escherichia coli DH5 $\alpha$. The plasmid DNA was extracted from the cultures using the QIAGEN miniprep kit, quantified by spectrometry (NanoDrop 2000 , Thermo Scientific), visualized in $0.7 \%$ agarose gel, and stored at $-20^{\circ} \mathrm{C}$ until use.

C. burnetii propagation in axenic culture. The C. burnetii strain Henzerling RSA 331 in phase II, obtained by passaging in embryonated hen's eggs in the presence of increasing doses of chlortetracycline (Brezina et al., 1975), was used in this study. The cells were propagated in ACCM-2 axenic medium at $\mathrm{pH} 4.75$ as described previously (Omsland et al., 2009; Stead et al., 2013). A volume of $40 \mathrm{ml}$ of medium was inoculated with a 3-day-old cell culture seed containing $1 \times 10^{9}$ cells as quantified by qPCR. The bacterium was propagated for 6 days in vented $75 \mathrm{~cm}^{3}$ culture flask at $37^{\circ} \mathrm{C}$ under the atmosphere of $2.5 \% \mathrm{O}_{2}$ and $5 \% \mathrm{CO}_{2}$ using Galaxy-170R incubator (New Brunswick Scientific). For the growth kinetic experiments, the cells were harvested daily with the sample taken immediately after inoculation considered 0 days post-inoculation (dpi). To the treated culture, sterile doxycycline $\left(1 \mathrm{mg} / \mathrm{ml}\right.$ in milliQ $\mathrm{H}_{2} 0$, ) was added every 24 hours to a final concentration of $0.5 \mu \mathrm{g} / \mathrm{ml}$. Finally, the bacterial cells were recovered by centrifugation at 19,000 $\mathrm{x} g$ in an Eppendorf 5810 centrifuge and washed twice with phosphate buffer saline before further analyses. A defined volume of various dilutions of C. burnetii cultures was used for direct counting using the Live/Dead BacLight bacterial viability kit (Thermo Fisher Scientific) following the manufacturer's recommendations.

DNA and RNA purification and cDNA synthesis. The DNA and RNA were extracted from the cellular pellet using the AllPrepDNA/ RNA/Proteins kit from QIAGEN. The extracted RNA was treated at $37^{\circ} \mathrm{C}$ for $8 \mathrm{~h}$ with RQ1 RNA-free DNAase (Promega) to eliminate possible contamination with genomic DNA according to the manufacturer's instructions. The reaction was stopped by DNAase inactivation reagent for $10 \mathrm{~min}$, then cleaned by RNeasy minElute cleanup kit from QIAGEN and quantified by spectrometry (NanoDrop 2000, Thermo Scientific). The presence of contaminating DNA was evaluated by PCR amplification of dotA-fragment gene 
using the primers dotA30/31 according to the conditions described above. The absence of $300 \mathrm{~kb}$ amplicon was verified by $1 \%$ agarose gel electrophoresis. Then, aliquots of $1 \mu \mathrm{g}$ of RNA were reverse transcribed using First-Strand cDNA synthesis kit (Thermo Scientific) in accordance with manufacturer's instructions. The resulted single-stranded cDNA was stored at $-20^{\circ} \mathrm{C}$ until use.

Real-time PCR ( $q P C R)$ and standard curve construction. Five replicative runs of a real-time PCR (qPCR) with seven tenfold dilutions of the plasmid $\mathrm{pCB}$-dotA were performed. The number of plasmid copies per nanogram of DNA mass were calculated using the equation: $(n \times M W)^{-1} \times\left(\mathrm{N} \times 10^{-9}\right)$, where $n$ is the number of base pairs of the plasmid (4,191 bp), $M W$ is the molecular weight per base pair $(649 \mathrm{~g} / \mathrm{mol})$ and $\mathrm{N}$ is the Avogadro constant $\left(6.022 \times 10^{23}\right.$ molecules per mol). For the calibration curve construction, aliquots of the plasmid in dilutions ranging from $3 \times 10^{1}$ to $3 \times 10^{8}$ copy numbers were applied. The qPCR assay was based on the Taq Man chemistry using the primers dotAF (5'-GCGCAATACGCTCAATCACA-3'), dotAR (5'-CCATGGCCCCAATTCTCTT-3') and QdotA (5'-Fam-CCGGAGATACCGGCGGTGGG-Tamra-3') described by Coleman et al. (2004). The reaction was executed in the CFX96 Touch Real-Time PCR Detection System (BioRad) utilizing the Scientific Maxima Probe/ROX qPCR Master Mix (Thermo Scientific). The PCR cycle consisted of initial denaturation at $95^{\circ} \mathrm{C}$ for $10 \mathrm{~min}, 40 \mathrm{cycles}$ of denaturation at $95^{\circ} \mathrm{C}$ for $30 \mathrm{~s}$, annealing at $60^{\circ} \mathrm{C}$ for $1 \mathrm{~min}$. For the standard curves, the threshold cycle $(\mathrm{Cq})$ values were calculated using the CFX Maestro Software provided by the qPCR equipment manufacturer.

$Q$ uantitative reverse transcription polymerase chain reaction ( $R T$ qPCR) of C. burnetii cells. The PCR reaction mixture consisted of $20 \mathrm{pg}$ aliquot of DNA isolated from the harvested cells at specific time intervals from the day of inoculation or $25 \mathrm{ng}$ of cDNA (obtained from $5 \mu$ of 1:100 dilution in water of cDNA synthesized from $1 \mu \mathrm{g}$ of RNA), $100 \mathrm{nmol} / \mathrm{l}$ of each qPCR primer, $0.8 \mu \mathrm{mol} / \mathrm{l}$ of probe, $12.5 \mu \mathrm{l}$ of Scientific Maxima Probe/ROX qPCR Master Mix (Thermo Scientific) in DNAse- and RNAse-free water added to a final volume of $25 \mu$ l. Since only a single copy of the $\operatorname{dot} A$ gene is present in C. burnetii genome, the genome equivalents (GE) were calculated using the equation: $g \times m$, where $g$ is the number of genomes per nanogram resulting from the real-time PCR calculated by extrapolation from the standard curve and $m$ is the total amount of respective DNA or CDNA extracted from the sample. The specificity test was performed with $20 \mathrm{ng}$ of DNA from either C. burnetii strain Henzerling RSA 331 in phase II, tolerant to chlortetracycline (Brezina et al., 1975), DNA from C. burnetii strain Scurry in phase I; NCBItxid:1321948 (Samuel et al., 1985), Rickettsia akari strain MK (ATC VR-148), Rickettsia slovaca strain 13-B; NCBItxid:941638 and Rickettsia conorii strain 7; NCBItxid:272944 obtained from the strain collection of the Laboratory for Diagnosis and Prevention of Rickettsial and Chlamydial Infections, Department of Rickettsiology, Institute of Virology, Biomedical Research Center, Slovak Academy of Sciences, Bratislava, Slovak Republic. All the real-time PCR products were analyzed by electrophoresis in $2 \%$ agarose gel.

\section{Results and Discussion}

During the last decades, several qPCR-based diagnostic assays were developed to detect C. burnetii DNA from cell cultures or clinical samples. The prerequisite for such a diagnostic is a target sequence that is specific for C. burnetii to exclude false positive results with other organisms and also conserved in all isolates to prevent false negative reactions. The target sequences used so far include the plasmid genes (QpH1, QpRS) (Willems et al., 1993), the singular chromosomal genes like com1, htpB, (Seshadri et al., 2003), icd (Klee et al., 2006), icmX, icm W, icm V, icmT, $\operatorname{dot} B$ (Morgan et al., 2010), dotA (Omsland et al., 2009; Cockrell et al., 2017), gyrA gene (Pradeep et al., 2017) or the transposase gene of insertion element IS1111, which is present in the genome of C. burnetii in multiple copies. Although qPCR quantification of the C. burnetii cells is very sensitive due to the presence of a multicopy number of this insertion element in the genome, the number of copies of this element in different isolates seem to be highly variable, which makes the quantification difficult (Klee et al., 2006).

\section{Plasmid construction}

With the aim to assess viable cell counts, we designed and evaluated an RT-qPCR assay that targeted the singular dotA gene (CBU1648) in the C. burnetii genome that is conserved among the known isolates (Segal et al., 2005). This gene is encoding an integral cytoplasmic transmembrane protein with eight membrane-spanning domains that form a pore for transport of effectors into the host cytoplasm during infection (Luedtke et al., 2017). The protein is a structural component of the type IVB secretion system (T4SS), the machinery that is evolutionarily related to bacterial conjugation. The system facilitates the transport of effector molecules (nucleic acids or proteins) into the interior of host cells with the aim to potentiate the bacterial survival and virulence (Luedtke et al., 2017). The plasmid pCB-dotA employed in the assay was assembled using a $291 \mathrm{bp}$ fragment from $C$. burnetii Nine Mile dotA gene (Fig. 1a). This gene has shown a constant relative expression level during morphological differentiation of C. burnetii (Coleman et al., 2004).

Determination of reproducibility, precision, specificity, and efficiency of the qPCR assay

To determine whether false positive reactions may occur with the selected $\operatorname{dot} A$ fragment, identical qPCR reactions were performed with DNAs isolated from purified bacterial species $R$. akari, $R$. slovaca, $R$. conorii, C. burnetii strain Scurry in phase I, and C. burnetii strain Henzerling RSA 331 in phase II. The product of the $\operatorname{dot} A$ qPCR assay was then evaluated by agarose gel electrophoresis (Fig. 1b). A single 
(a)

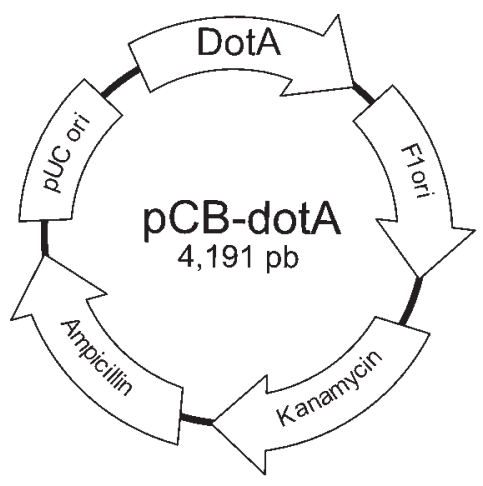

(c)

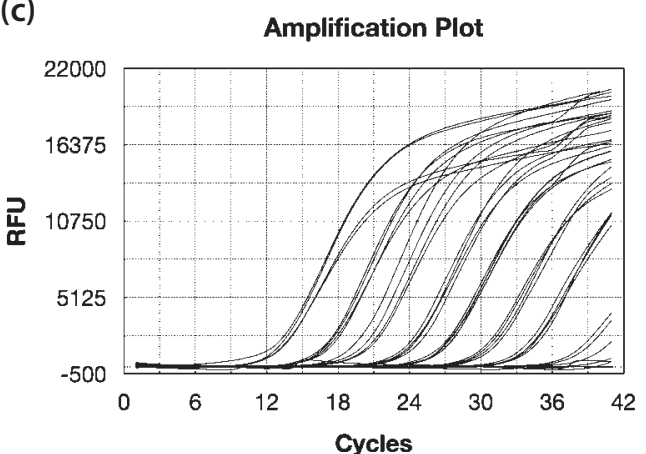

(b)

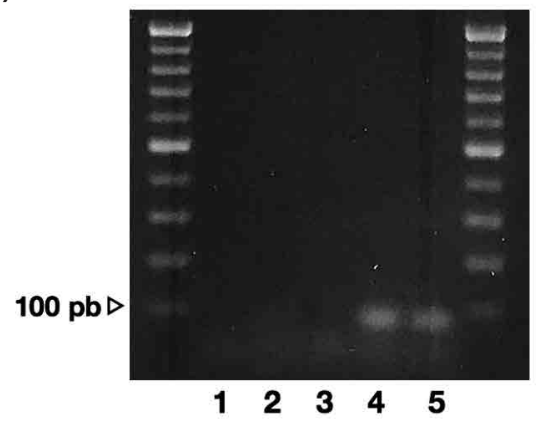

(d)

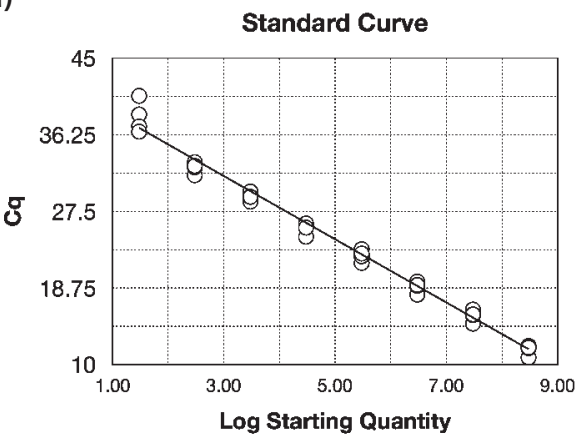

Fig. 1

Title

(a) Map of the pCB-dotA plasmid. (b) amplification of C. burnetii dotA gene. Ten microliters of the products after RT-PCR were resolved in 2\% agarose gel. From left to right: Molecular weight marker and samples from 1) Rickettsia akari, 2) Rickettsia slovaca, 3) Rickettsia conorii, 4) C. burnetii strain Henzerling RSA 331 and 5) C. burnetii strain Scurry. (c) Amplification plots of dotA standards from $3 \times 10^{1}$ to $3 \times 10^{8}$ copies. (d) Standard curve generated from the Cq values calculated by the CFX Manager Software.

band of about $100 \mathrm{bp}$ was detected in C. burnetii samples. This band was absent from all other tested related bacterial species, which confirms the specificity of the target sequence.

A set of qPCR assays in a tenfold dilution series of the plasmid pCB-dotA were performed. Representative amplification curves are shown in Fig. 1c. Table 1 summarizes the average from five replicate runs of the eight independent

Table 1. Summary of the average of PCR runs performed with eight tenfold dilutions of the plasmid pCB2-dotA

\begin{tabular}{cccc}
\hline Dilution & Mean Cq & SD $^{\mathbf{a}}$ & $\mathbf{C V}^{\mathbf{b}}$ \% \\
\hline $3 \times 10^{8}$ & 11.91 & 0.74 & 6.23 \\
$3 \times 10^{7}$ & 15.83 & 0.82 & 5.16 \\
$3 \times 10^{6}$ & 19.13 & 0.80 & 4.18 \\
$3 \times 10^{5}$ & 22.76 & 0.90 & 3.96 \\
$3 \times 10^{4}$ & 25.72 & 0.72 & 2.81 \\
$3 \times 10^{3}$ & 29.45 & 0.76 & 2.57 \\
$3 \times 10^{2}$ & 32.78 & 0.85 & 2.58 \\
$3 \times 10^{1}$ & 38.27 & 1.81 & 4.73 \\
\hline
\end{tabular}

${ }^{a} \mathrm{SD}$ standard deviation of five PCR runs. ${ }^{\mathrm{b}} \mathrm{CV}$ coefficient of variation. tenfold dilutions of the plasmid. The results showed that the quantification was linear over a range of $3 \times 10^{1}$ to $3 \times 10^{8}$ plasmid copy numbers (Fig. 1d). The calculated average value of the slopes from the standard series was -3.44 with a standard deviation of the mean (SD) of \pm 0.04 , which indicated a mean assay efficiency of about $95.14 \%$ with a SD of $\pm 1.38 \%$ according to the equation $\mathrm{E}=10^{-1 / \mathrm{s}}-1$, where $E$ is the run efficiency and $s$ refers to the slope of the standard curve (Fink et al., 1998). Furthermore, low variability, with a Coefficient of Variation (CV) ranging from $2.58 \%$ to $6.23 \%$, indicated efficient reproducibility of the assay. Using this approach, we determined that the minimal number of copy numbers that could be detected with a $97.42 \%$ probability was $3 \times 10^{1}$, while in the upper level $3 \times 10^{8}$ copies can be detected at 93.77\% feasibility.

Assessment of the viable cell counts of C. burnetii propagated in axenic culture.

evaluate the analytical performance of the proposed assay, we measured the growth of C. burnetii Henzerling RSA 331 
○ Antibiotic Free (AF) $\Delta$ Antibiotic Treated (AT)

(a)

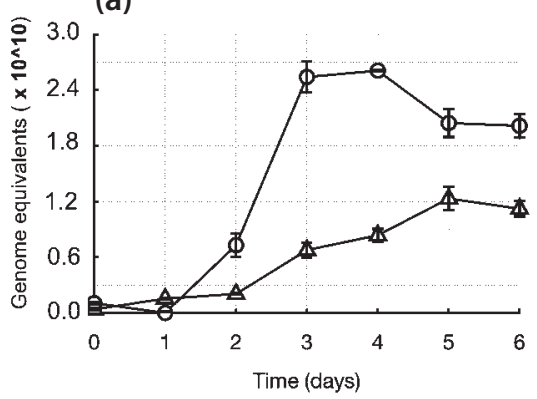

(b)

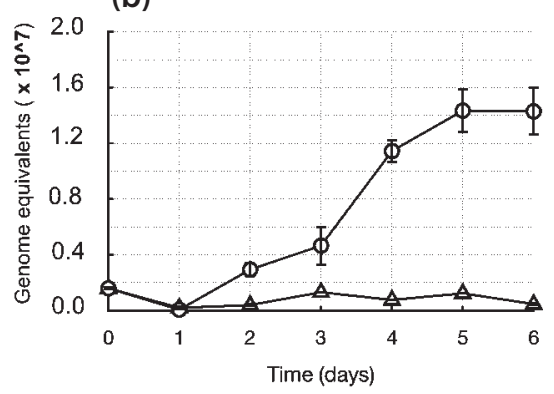

Fig. 2

Growth kinetics of C. burnetii

The graphics show the results obtained from quantification of cell amounts growing in antibiotic-free (AF) and antibiotic-treated media (AT) at $0.5 \mathrm{mg} / \mathrm{ml}$, employing (a) DNA and (b) cDNA. The bars represent the standard deviation of the mean for three replicates.

strain tolerant to chlortetracycline in axenic culture under absence and presence of $0.5 \mu \mathrm{g} / \mathrm{ml}$ doxycycline for 6 days. Hence it was assumed that only a single copy of the $\operatorname{dot} A$ gene is present in the C. burnetii genome and only one copy of the chromosome is present per bacterial cell, which can be expected for a slow-growing bacterium like Coxiella; the number of GE based on $\operatorname{dot} A$ quantification should be comparable to the number of bacterial cells. Thus, we determined the GE in the culture over a particular period of time and plotted a growth curve (Fig. 2). Analysis of the data showed decreased growth of $C$. burnetii in the presence of doxycycline. While the cell count was only 0.002 times greater in the antibiotic-free (AF) compared to antibiotic-treated culture (AT) at $1 \mathrm{dpi}$, this difference changed significantly over the time. At 3 dpi, the GE of AF vs. AT culture was 3,74 times higher, probably due to the susceptibility of the bacterial culture to doxycycline (Yeaman et al., 1989; Gikas et al., 2001). The discrepancy in GE level decreased to 2.82 at day 6 as a result of decreased growth rate due to limited nutrient supply present in the medium (Fig. 2a).

As the correlation between the presence of DNA and cell viability is ambiguous (Keer and Birch, 2003), we performed RT-qPCR utilizing cDNA synthesized from RNA. Similarly as above, the GE number estimated for AF culture was only a little higher ( 0.32 times) as for AT at $1 \mathrm{dpi}$. This difference, however, continually increased over the time due to sustained growth in AF culture, where the cells were propagated steadily up to day 5 . On the other hand, bacteria in the doxycycline-treated medium grew slowly until day 3 when they reached the maximum and remained constant up to the end of the kinetic plot. The highest difference was observed at 6 dpi when the GE number in the AF culture was 30.79 times higher than the AT. The slight increase in the cell count in AT culture followed by slow reduction is probably associated with the bacteriostatic effect of doxycycline and moderate adaptation of the bacterium to the agent. This observation indicates that a small population of C. burnetii preserves the ability to reproduce while the remaining cells are either dead or have inhibited growth as a consequence of antibiotic treatment. To assess whether the cell densities quantified by qPCR provide a reliable result, we validated them by commercial Live/Dead BacLight bacterial viability kit. Indeed, the $\operatorname{dot} A$ copies were correlated well with the numbers of particles determined microscopically (data not shown). Moreover, a high percentage of cells with a compromised membrane that is considered to be dead or dying was confirmed on the last days of antibiotic- treated culture.

\section{Conclusion}

We validated a TaqMan-based qPCR assay targeting the fragment of $C$. burnetii $\operatorname{dot} A$ gene. The assay was shown to be specific, sensitive and efficiently reproducible. Copy numbers of the designed plasmid were quantified in a range from $3 \times 10^{1}$ to $3 \times 10^{8}$ bacteria with a high reliability, allowing the monitoring of behavior of the cell culture exposed to doxycycline. In this regard, the capacity to evaluate the effect of different agents could be crucial for the development of studies focused not only in the basic knowledge of the bacteria but also on the development of alternative therapies to contend with this dangerous pathogen.

Acknowledgments. This work was supported by the following grants Nos. 2/0068/18, 2/0139/16 and 2/0144/15 from the Scientific Grant Agency of the Ministry of Education of the Slovak Republic, the Slovak Research and Development Agency (APVV-14-0474), the Research Grant (2014) of the European Society of Clinical Microbiology and Infectious Diseases (ESCMID) to (GFR), and the grant No. 26240220096 of the Research \& Development Op- 
erational Programme funded by the European Regional Development Fund.

\section{References}

Babudieri B (1959): Q fever: a zoonosis. Adv. Vet. Sci. 5, 101. Baca OG, Paretsky D (1983): Q fever and Coxiella burnetii: a model for host-parasite interactions. Microbiol. Rev. 47, 127-149.

Bustin S, Nolan T (2009): Analysis of mRNA expression by Real Time-PCR. In Logan J, Edwards K, Sanders N (Ed.): Real-time PCR: Current Technology and Applications. Horizon Scientific Press, pp. 111-135.

Coleman SA, Fischer ER, Howe D, Mead DJ, Heinzen RA (2004): Temporal analysis of Coxiella burnetii morphological differentiation. J. Bacteriol. 186, 7344-7352. https://doi. org/10.1128/JB.186.21.7344-7352.2004

Cockrell DC, Long CM, Robertson SJ, Shannon JG, Miller HE, Myers L, Larson CL, Starr T, Beare PA, Heizen RA (2017): Robust growth of avirulent phase II Coxiella burnetii in bone marrow-derived murine macrophages. PLoS One 12, e0173528. https://doi.org/10.1371/journal. pone. 0173528

Gikas A, Spyridaki I, Scoulica E, Psaroulaki A, Tselentis Y (2001): In vitro susceptibility of Coxiella burnetii to linezolid in comparison with its susceptibilities to quinolones, doxycycline, and clarithromycin. Antimicrob. Agents Chemother. 45, 3276-3278. https://doi.org/10.1128/ AAC.45.11.3276-3278.2001

Hackstadt T, Peacock MG, Hitchcock PJ, Cole RL (1985): Lipopolysaccharide variation in Coxiella burnetii: intrastrain heterogeneity in structure and antigenicity. Infect. Immun. 48, 359-365.

Keer JT, Birch L (2003): Molecular methods for the assessment of bacterial viability. J. Microbiol. Methods 53, 175-183. https://doi.org/10.1016/S0167-7012(03)00025-3

Klee SR, Tyczka J, Ellerbrok H, Franz T, Linke S, Baljer G, Appel B (2006): Highly sensitive real-time PCR for specific detection and quantification of Coxiella burnetii. BMC Microbiol. 6, 2. https://doi.org/10.1186/1471-2180-6-2

Luedtke BE, Mahapatra S, Lutter EI, Shaw EI (2017): The Coxiella burnetii type IVB secretion system (T4BSS) component DotA is released/secreted during infection of host cells and during in vitro growth in a T4BSS-dependent manner. Pathog. Dis. 75, ftx047. https://doi.org/10.1093/ femspd/ftx047

Mannhalter C, Koizar D, Mitterbauer G (2000): Evaluation of RNA isolation methods and reference genes for RT-PCR analyses of rare target RNA. Clin. Chem. Lab. Med. 38, 171-177. https://doi.org/10.1515/CCLM.2000.026

Marrie TJ (1990): Epidemiology of Q fever. In Q fever: The disease. Boca Raton, FL, CRC. 1, 21.

Masters CI, Shallcross JA, Mackey BM (1994): Effect of stress treatments on the detection of Listeria monocytogenes and enterotoxigenic Escherichia coli by the polymerase chain reaction. J. Appl. Bacteriol. 77, 73-79. https://doi. org/10.1111/j.1365-2672.1994.tb03047.x
Maurin M, Raoult D (1999): Q fever. Clin. Microbiol. Rev. 12, 518-553. https://doi.org/10.1128/CMR.12.4.518

Morgan JK, Luedtke BE, Thompson HA, Shaw EI (2010): Coxiella burnetii type IVB secretion system Region I genes are expressed early during infection of host cells. FEMS microbiol. Lett. 311, 61-69. https://doi.org/10.1111/j.15746968.2010.02072.x

Omsland A, Beare PA, Hill J, Cockrell DC, Howe D, Hansen B, Samuel JE, Heinzen RA (2011): Isolation from animal tissue and genetic transformation of Coxiella burnetii are facilitated by an improved axenic growth medium. Appl. Environ. Microbiol. 77, 3720-3725. https://doi. org/10.1128/AEM.02826-10

Omsland A, Cockrell DC, Howe D, Fischer ER, Virtaneva K, Sturdevant DE, Porcella SF, Heinzen RA (2009): Host cellfree growth of the $\mathrm{Q}$ fever bacterium Coxiella burnetii. Proc. Natl. Acad. Sci. USA 106, 4430-4434. https://doi. org/10.1073/pnas.0812074106

Pradeep J, Stephen S, Ambroise S, Gunasekaran D (2017): Diagnosis of Acute Q Fever by Detection of Coxiella burnetii DNA using Real-Time PCR, Employing a Commercial Genesig Easy Kit. J. Clin. Diagn. Res. 11, DC10-DC13. https://doi. org/10.7860/JCDR/2017/31005.10606

Segal G, Feldman M, Zusman T (2005): The Icm/Dot type-IV secretion systems of Legionella pneumophila and Coxiella burnetii. FEMS Microbiol. Rev. 29, 65-81. https://doi. org/10.1016/j.femsre.2004.07.001

Seshadri R, Paulsen IT, Eisen JA, Read TD, Nelson KE, Nelson WC, Ward NL, Tettelin H, Davidsen TM, Beanan MJ, Deboy RT, Daugherty SC, Brinkac LM, Madupu R, Dodson RJ, Khouri HM, Lee KH, Carty HA, Scanlan D, Heinzen RA, Thompson HA, Samuel JE, Fraser CM, Heidelberg JF (2003): Complete genome sequence of the Q-fever pathogen Coxiella burnetii. Proc. Natl. Acad. Sci. USA 100, 5455-5460. https://doi.org/10.1073/pnas.0931379100

Raoult D, Houpikian P, Tissot Dupont H, Riss JM, Arditi-Djiane J, Brouqui P (1999): Treatment of Q fever endocarditis: comparison of 2 regimens containing doxycycline and ofloxacin or hydroxychloroquine. Arch. Intern. Med. 159, 167-173. https://doi.org/10.1001/archinte.159.2.167

Samuel JE, Frazier ME, Mallavia LP (1985): Correlation of plasmid type and disease caused by Coxiella burnetii. Infect. Immun. 49, 775-779.

Stead CM, Omsland A, Beare PA, Sandoz KM, Heinzen RA (2013): Sec-mediated secretion by Coxiella burnetii. BMC Microbiol. 13, 222. https://doi.org/10.1186/1471-2180-13-222

Wike DA, Tallent G, Peacock MG, Ormsbee RA (1972): Studies of the rickettsial plaque assay technique. Infect. Immun. $5,715-722$.

Willems H, Thiele D, Krauss H (1993): Plasmid-based differentiation and detection of Coxiella burnetii in clinical samples. Eur. J. Epidemiol. 9, 411-418. https://doi.org/10.1007/ $\underline{B F 00157399}$

Yeaman MR, Roman MJ, Baca OG (1989): Antibiotic susceptibilities of two Coxiella burnetii isolates implicated in distinct clinical syndromes. Antimicrob. Agents Chemother. 33, 1052-1057. https://doi.org/10.1128/AAC.33.7.1052 